\title{
An Heuristic Approach to Solving the one-to-one Pickup and Delivery Problem with Three- dimensional Loading Constraints
}

\author{
Rémy Dupas \\ Univ. Bordeaux, CNRS, IMS, UMR 5218, 33405 Talence, France \\ E-mail: remy.dupas@gmail.com \\ Igor Grebennik \\ Kharkiv National University of Radio Electronics, Kharkiv, Ukraine \\ E-mail: igorgrebennik@gmail.com \\ Oleksandr Lytvynenko and Oleksij Baranov \\ Kharkiv National University of Radio Electronics, Kharkiv, Ukraine \\ E-mail: litvinenko1706@gmail.com, aleksey.baranov@gmail.com
}

Received: 17 May 2017; Accepted: 01 July 2017; Published: 08 October 2017

\begin{abstract}
A mathematical model and a solving strategy for the Pickup and Delivery Problem with threedimensional loading constraints regarding a combinatorial configuration instead of a traditional approach that utilizes Boolean variables is proposed. A traditional one-to-one Pickup and Delivery Problem in a combination with a problem of packing transported items into vehicles by means of the proposed combinatorial generation algorithm is solved.
\end{abstract}

Index Terms-Pickup and Delivery problem, vehicle routing, 3D loading constraints, combinatorial configuration, generation, packing of parallelepipeds.

\section{INTRODUCTION}

A vehicle routing problem (VRP) plays an important role in the logistics management. A wide variety of the vehicle routing problems has been studied lately [1-3]. Different classes of the vehicle routing problem describe various practical situations, but they are mostly focused on a common problem - an efficient use of a set of vehicles that must serve customers' orders.

In addition to routing vehicles, real-world transportation companies also require solving a problem of loading vehicles which means that it is not sufficient enough only to decide how to route vehicles, but also how to load cargos to them. Such an integrated problem was introduced in [4] for the first time and it was called as the "capacitated vehicle routing problem" (CVRP) with three-dimensional (3D) loading constraints (3LCVRP). In 3L-CVRP, every customer requires transporting one or a few parallelepipeds (or boxes) where each one is represented by a $3 \mathrm{D}$ rectangular loading space and its weight (in contrast to the traditional
CVRP, where an only weight is given). Surveys [5] and [6] show a recent state of the art for solving the integrated vehicle routing and loading problems.

One of the most popular VRP models is the Pickup and Delivery Problem (PDP) [7-10], where every customer has to pick up some item/items at one location and to deliver it/them to another location. The Pickup and Delivery Problem arises naturally in several contexts such as urban courier services and door-to-door transportation systems [11].

The Pickup and Delivery Problem (PDP) is about routing a set of vehicles in order to serve a set of transportation requests between given origins (pickup points) and destinations (delivery points). Every route should start and finish at a pre-defined depot and satisfy pairing and precedence constraints: the origin (a pickup point) should precede the destination (a delivery point), and every pickup-delivery pair should be visited by the same vehicle [11]. There are a lot of additional constraints on PDP such as time windows [7, 12], time constraints related to vehicles availability [10], etc.

A lot of heuristics and metaheuristics were used for solving PDP: the reactive tabu search [13], the tabu embedded simulated annealing [14], the squeaky wheel optimization [15], the grouping genetic algorithm [16], the construction heuristic [17], the hybrid algorithm (the simulated annealing and the large neighborhood search) [18], the adaptive large neighborhood search, the indirect local search with greedy decoding [19], and the guided ejection search [20] etc.

In case of the vehicle routing problem, solving the Pickup and Delivery Problems in the real-world applications also demands taking the loading constraints into account. Articles which solve PDP consider such constraints as LIFO [21] or FIFO [22] buffers, or as the 2D or 3D loading constraints [23-26]. 
Despite a wide variety of articles dedicated to PDP, it is hard or impossible to regulate a balance between a solution time and a result's precision in most of the solution algorithms. An objective function and all limitations in PDP are usually described as inequalities with the help of Boolean variables.

In this article, a mathematical model for the Pickup and Delivery Problem with the 3D loading constraints which utilizes combinatorial sets instead of commonly used Boolean variables is provided. The combinatorial generation algorithm for solving the described problem is also given. An advantage of the described algorithm is its ability to balance between the quality and the time of solution. The Given solution algorithm produces quite good results in a reasonable time.

The paper is organized as follows. Section II describes a mathematical model. Section III gives basic details of the decision strategy. Section IV demonstrates the solution algorithm for a lower level. Section V gives explanations to computational experiments. Conclusions are given in Section VI.

\section{THE MATHEMATICAL MODEL}

\section{A. The problem formulation}

We are considering the traditional Pickup and Delivery Problem (PDP) [27], one-to-one, a symmetric case, i.e. every $\operatorname{arc}(i, j)$ is equal to the $\operatorname{arc}(j, i)$ and can be replaced by one edge. The Pickup and Delivery Problem is modeled on a complete graph $G=(V, A)$ where $V$ is a set of all vertices, $V=\{0,1, \ldots, 2 n+1\}$, where 0 and $2 n+1$ denote a depot and $A$ is a set of all the arcs.

There are $v$ identical vehicles available; each vehicle has a loading space in the form of a parallelepiped (defined by a width $W$, a height $H$ and a length $L$ ) and a weight capacity $Q$. Every transportation request $i \in J_{n}$, $J_{n}=\{1,2, \ldots, n\}$ requires the pickup or delivery of one three-dimensional item having a width $w_{i}$, a height $h_{i}$ and a length $l_{i}$ with a total weight $q_{i}$ ( $q_{i}$ is positive for pickups and is negative for delivery points). We assume that all the items are rectangular boxes.

There are some constraints on the loading items of a vehicle (the $3 D$ constraints):

1. Inside a vehicle, the items can only be placed orthogonally; however, they can be rotated by $90^{\circ}$ in the width-length plane.

2. The stability constraint: every transported item should be placed on a vehicle's floor; it can be also placed on top of another item. In such case, the item should be completely supported by the one below, i.e. we do not allow any part of the item to be in limbo.

3. The blocking constraint: we should ensure that items can be easily unloaded in their delivery point which means that when a delivery point is visited, two conditions should be satisfied:

- $\quad$ an item to be unloaded should not be stacked beneath other items in the vehicle. An item $\mathrm{A}$ is beneath an item B if the interior of the projections of their bases to the vehicle's floor intersects, and the top of $\mathrm{A}$ is not higher than the bottom of $\mathrm{B}$ in a vertical direction;

- the unloaded item should not also be blocked by other clients' items that will be visited later. The item is also blocked if it overlaps any item of a next client when it is moved along the $\mathrm{L}$ axis towards a rear door.

The objective is to find a set of at most $v$ routes (one per a vehicle) such as:

1. Every route begins at the depot and after all clients have been visited ends at the depot.

2. Every client (i.e. a pair of pickup and delivery points) is served by the same vehicle.

3. A total weight of transported items does not exceed a vehicle's capacity.

4. Items are packed in a vehicle according to the 3D constraints.

5. A total cost of all routes is minimized.

\section{B. Designations}

$P$ denotes a set of pickup vertexes, $P=\{1,2, \ldots, n\}$;

$\mathrm{D}$ signifies a set of delivery vertexes, $D=\{n+1, n+2, \ldots, 2 n\}$;

$q_{i}$ marks a vehicle's load at the vertex $i ; q_{i}$ is positive at pickup nodes $i=\{1,2, \ldots, n\}$; it is negative at delivery nodes $i=\{n+1, n+2, \ldots, 2 n\}$;

$w_{i}, h_{i}, l_{i}$ signify orthogonal dimensions (a width, a height and a length) of the item at the vertex $i, i \in J_{2 n}$;

$v$ is a number of vehicles;

$Q$ determines a capacity of a single vehicle (all vehicles have the same capacity);

$W, H, L$ are orthogonal dimensions (a width, a height and a length) of every loading space respectively,

$C$ stands for a set of the pickup-delivery pairs, $C=\left\{\left(p_{i}, d_{i}\right)\right\}, p_{i} \in P, d_{i} \in D, d_{i}=p_{i}+n, i \in J_{n} ;$

$C_{1}, C_{2}, \ldots, C_{v}$ define a partition of $C: C=\bigcup_{j=1}^{v} C_{j}$, $C_{i} \cap C_{j}=\varnothing \quad, \quad i \in J_{v}, j \in J_{v} \quad$; each subset $C_{j}$ corresponds to a vehicle $j$ that serves this set of clients, $j \in J_{v}$,

$n_{j}=\operatorname{Card} C_{j}, j \in J_{v}, \sum_{j=1}^{v} n_{j}=n ;$

$c(i, j)$ is a cost of a traversing edge $(i, j)$;

$V_{j}=\left\{i_{1}^{j}, i_{2}^{j}, \ldots, i_{2 n_{j}}^{j}\right\}$ is a set of all pickup and delivery 
points included into $C_{j}$;

$P\left(V_{j}\right)$ stands for a set of permutations of elements from $V_{j}$ that describes all possible paths of the vehicle $j$;

$Q\left(i_{k}^{j}\right)$ denotes a current load of the vehicle $j$ at a moment of arrival to the vertex $i_{k}^{j}, k \in J_{2 n_{j}}$;

$u_{0}^{j}=\left(x_{0}^{j}, y_{0}^{j}, z_{0}^{j}\right)$ determines coordinates of a pole of the placement area in the vehicle $j$.

\section{Decision variables of the problem}

$U=\left(U^{1}, U^{2}, \ldots, U^{\mu}\right), U^{j}=\left(u_{1}^{j}, u_{2}^{j}, \ldots, u_{n_{j}}^{j}\right)$ where

$u_{i}^{j}=\left(x_{i}^{j}, y_{i}^{j}, z_{i}^{j}\right)$ stands for coordinates of the pole of the item $i$ in the vehicle $j$;

$\pi^{j} \in P\left(V_{j}\right)$ is a route of the vehicle $j$;

$v_{i}^{j}=\left(l w_{i}, h_{i}\right), l w_{i} \in\left\{\left(l_{i}, w_{i}\right),\left(w_{i}, l_{i}\right)\right\}$ marks orientation of the item $i$ in the vehicle $j, i \in V_{j}, j \in J_{\mu}$.

For $n$ transported items

$$
\begin{gathered}
\Pi_{i}=\left\{x \in R^{3}: x=\left(x_{1}, x_{2}, x_{3}\right) \mid 0 \leq x_{1} \leq l_{i},\right. \\
\left.0 \leq x_{2} \leq w_{i}, 0 \leq x_{3} \leq h_{i}\right\}, i \in J_{n},
\end{gathered}
$$

we have $v$ identical placement areas $D_{j}, j \in J_{v}$ :

$$
\begin{gathered}
D_{j}=\left\{x \in R^{3}: x=\left(x_{1}, x_{2}, x_{3}\right) \mid 0 \leq x_{1} \leq L,\right. \\
\left.0 \leq x_{2} \leq W, 0 \leq x_{3} \leq H\right\} .
\end{gathered}
$$

\section{D. $\Phi$-functions}

$\Phi$-functions [28] make it possible to describe formally conditions of the mutual non-intersection for two parallelepipeds and the condition for correct placement of parallelepipeds in a placement area [29].

To describe the 3D constraints, we use two $\Phi$ functions:

$\Phi_{i l}^{j}\left(u_{i}^{j}, u_{m}^{j}, v_{i}^{j}, v_{m}^{j}\right)$ is used for checking that the item $i$ (which is determined by coordinates of a pole $u_{i}^{j}$ and an orientation $v_{i}^{j}$ ) does not intersect with an item $m$ (which is determined by coordinates of a pole $u_{m}^{j}$ and an orientation $v_{m}^{j}$ );

$\Phi_{0 m}^{j}\left(u_{0}^{j}, u_{m}^{j}, v_{m}^{j}\right)$ is used for checking that the item $m$ can be correctly placed into the placement area $D_{j}$ (which is determined by $W$ (a width), $H$ (a height) and $L$ (a length) of the vehicle's loading space).

$$
\begin{gathered}
\Phi_{i l}^{j}\left(u_{i}^{j}, u_{m}^{j}, v_{i}^{j}, v_{m}^{j}\right)=\max \left\{x_{m}^{j}-x_{i}^{j}-v_{1 i},\right. \\
-x_{m}^{j}+x_{i}^{j}-v_{1 m}, y_{m}^{j}-y_{i}^{j}-v_{2 i},-y_{m}^{j}+y_{i}^{j}-v_{2 m},
\end{gathered}
$$

$$
\begin{gathered}
\left.z_{m}^{j}-z_{i}^{j}-v_{3 i},-z_{m}^{j}+z_{i}^{j}-v_{3 m}\right\} \\
\Phi_{0 m}^{j}\left(u_{0}^{j}, u_{m}^{j}, v_{m}^{j}\right)=\min \left\{x_{m}^{j}-x_{0}^{j},-x_{m}^{j}+x_{0}^{j}+L-v_{1 m},\right. \\
y_{m}^{j}-y_{0}^{j},-y_{m}^{j}+y_{0}^{j}+W-v_{2 m}, z_{m}^{j}-z_{0}^{j}, \\
\left.-z_{m}^{j}+z_{0}^{j}+H-v_{3 m}\right\} .
\end{gathered}
$$

If $\Phi_{i m}^{j}\left(u_{i}^{j}, u_{m}^{j}, v_{i}^{j}, v_{m}^{j}\right) \geq 0$ for all $i, m \in J_{n}, i<m$, then there's no intersecting pair of items in a vehicle.

If $\Phi_{0 m}^{j}\left(u_{0}^{j}, u_{m}^{j}, v_{m}^{j}\right) \geq 0$ for all $m \in J_{n}$, then each item is placed correctly inside the vehicle's loading area.

Thus, items' placement in vehicles should be performed in such a way that the described $\Phi$-functions are positive.

E. An objective function and constraints

$$
\sum_{j=1}^{\mu}\left[c\left(0, i_{1}^{j}\right)+\sum_{k=1}^{2 n_{j}-1} c\left(i_{k}^{j}, i_{k+1}^{j}\right)+c\left(i_{2 n_{j}}^{j}, 2 n+1\right)\right] \rightarrow \min
$$

$$
Q\left(i_{k}^{j}\right)=\sum_{k=1}^{s} f\left(i_{k}^{j}\right) \leq Q, \quad \forall s \in J_{2 n_{j}}, j \in J_{\mu} ;
$$

$f(i)=\left\{\begin{array}{l}q_{i}, \quad \text { if } i<=n(\text { a vertex is a pickup }) ; \\ -q_{i}, \quad \text { if } i>n(a \text { vertex is a delivery })\end{array}\right.$

$\left\{\begin{array}{l}\Phi_{i m}^{j}\left(u_{i}^{j}, u_{m}^{j}, v_{i}^{j}, v_{m}^{j}\right) \geq 0, i, m \in J_{n}, i<m, \\ \Phi_{0 m}^{j}\left(u_{0}^{j}, u_{m}^{j}, v_{m}^{j}\right) \geq 0, m \in J_{n},\end{array}, j \in J_{\mu}\right.$.

Here $c\left(0, i_{1}^{j}\right)$ is a distance between the depot (a fictive vertex 0 ) and the first vertex visited by the vehicle $j$; $c\left(i_{2 n_{j}}^{j}, 2 n+1\right)$ is a distance between the last visited vertex and the depot (a fictive vertex $2 n+1$ ). It should be noted that the fictive vertexes 0 and $2 n+1$ designate the same depot.

\section{THE DECISION STRATEGY}

We propose a two-level strategy for solving the problem.

\section{A. An upper level-partitioning}

In the upper level, we are splitying a set $C$ into subsets (clusters) $C_{1}, C_{2}, \ldots, C_{v}$. Each cluster $C_{j}$ contains pickup-delivery pairs $\left(p_{i}, d_{i}\right)$ which are served by the vehicle $j$.

For solving the clustering [30] problem, we chose the simplest $k$-means algorithm [31-33]. The traditional kmeans algorithm deals with single points, but we want to make clusters of pairs $\left(p_{i}, d_{i}\right)$. We are substituting the 
pair $\left(p_{i}, d_{i}\right)$ with a single point $k_{i}$, which is a middle point between $p_{i}$ and $d_{i}$ :

$$
k_{i} \cdot x=\frac{p_{i} \cdot x+d_{i} \cdot x}{2}, k_{i} \cdot y=\frac{p_{i} \cdot y+d_{i} \cdot y}{2}
$$

where $x, y$ are coordinates of the points.

\section{B. A lower level-path constructing}

In the lower level, we are constructing a route for the vehicle $j$ for a single cluster $C_{j}$.

As mentioned above, a permutation $\pi^{j} \in P\left(V_{j}\right)$ describes a path of the vehicle $j$. The vehicle's route defines an order of items' loading and unloading to/from the vehicle $j$.

Every path $\pi^{j}$ should meet all the constraints described in Section 2. To describe items' rotations in the width-length plane, we substitute each element of $\pi^{j}$ (which is either a pickup point or a delivery one) by a vector $l w_{i} \in\left\{\left(l_{i}, w_{i}\right),\left(w_{i}, l_{i}\right)\right\}, i \in V_{j}$. This combinatorial permutation is called "a composition of permutations" [34].

So, to construct a route for the vehicle $j$, we should choose a permutation $\pi^{j} \in P\left(V_{j}\right)$ so that it minimizes the objective function (1). The algorithm's solution for this problem is described in the next section.

\section{SOLVING THE ALGORITHM FOR THE LOWER LEVEL}

\section{A. The exact solution}

To generate permutations $\pi^{j}$, we use the GenBase algorithm [35]. It is universal and can be used for generating a wide variety of different combinatorial sets.

Let us denote a path of the vehicle $j$ as $t=\pi^{j}$; let's also call first $i$ vertexes of a path $t$ as a partial path $t^{i}=\left(t_{1}, t_{2}, \ldots, t_{i}\right)$.

The GenBase algorithm is recursive: at every level $i \in J_{2 n-1}^{0}, J_{2 n-1}^{0}=\{0,1 \ldots 2 n-1\}$, it adds a successive vertex $t_{i+1}$ to the end of the current partial path $t^{i}=\left(t_{1}, t_{2}, \ldots, t_{i}\right)$ and obtains a new partial path $t^{i+1}=\left(t_{1}, t_{2}, \ldots, t_{i+1}\right)$ at the next level. At the level $i=2 n$, the algorithm produces a full path $t^{2 n}=t$.

Elements $t_{i+1}$ should meet the constraints specific to a particular combinatorial set. At every level $i \in J_{2 n-1}^{0}$, let us denote a tuple of all those elements as $F^{i}=\left(f_{1}, f_{2}, \ldots, f_{k}\right)$. So, for each $j \in J_{k}, J_{k}=\{1,2 \ldots k\}$, the GenBase algorithm adds a new element $t_{i+1}=f_{j}$ to the current partial path $t^{i}=\left(t_{1}, t_{2}, \ldots, t_{i}\right)$ and recursively calls itself with the new partial path $t^{i+1}=\left(t_{1}, t_{2}, \ldots, f_{j}\right)$.

To generate all the paths, the GenBase algorithm is called with an empty path $t^{0}=(\mathrm{)}$.

$$
\begin{aligned}
& \text { function GenBase }\left(t^{i}\right)\{ \\
& \text { if } i=2 n \text { then }\left\{\text { output }=\text { output } \cup t^{i} ; \text { exit; }\right\} \\
& \text { determine } F^{i} ; \\
& \text { for } j=1,2, \ldots,\left|F^{i}\right| \text { do } \\
& \quad \operatorname{GenBase}\left(t^{i+1}=\left(t_{1}, t_{2}, \ldots, t_{i}, f_{j}\right)\right) \text {; } \\
& \text { \} }
\end{aligned}
$$

For PDP paths, we have following constraints for a tuple $F^{i}$ :

1) vertexes in $t^{i}$ aren't duplicated

$$
t_{i+1} \neq t_{z}, z=1 \ldots i
$$

2) for every pickup-delivery pair, a vehicle should visit a pickup point before a corresponding delivery point:

$$
t_{i+1}>n \Rightarrow \exists z:\left(t_{i+1}-n\right)=t_{z} .
$$

For example, when $n=4$ (i.e. there are four pickupdelivery pairs), the delivery point 5 can be added to the path only if the corresponding pickup point 1 has already been added to the path;

3) the restriction (2) that limits the maximum vehicle's load should be satisfied;

4) if $t_{i+1}$ is a pickup, it means that a new item will be loaded into a vehicle, and we should check the 3Dconstraints. For this reason, the algorithm [29] should be used.

It should be noted that the algorithm [29] has an ability to rotate every item in the width-length plane for the optimal packing. Thus, vectors $l w_{i}, i \in V_{j}$, are determined.

The GenBase algorithm produces a recursive tree, where every node at intermediate levels $i<2 n-1$ is a partial path and nodes at the last level $i=2 n-1$ are full paths. At levels $i<2 n-1$, a tree node is expanded by adding a new node from $F^{i}$ to a partial path.

Example 1. Let's demonstrate how GenBase works while generating paths for $n=2$ (the vertexes 1 and 2 are pickups, and the vertexes 3 and 4 are deliveries).

At first, at the level $i=0, F^{0}$ consists only of pickup vertexes $F^{0}=(1,2)$. Each pickup is added to the end of a current empty path $t^{0}=()$ making a new path $\left(t^{1}=(1)\right.$ in the first case and $t^{1}=(2)$ in the second one). After that, GenBase is called recursively for each vertex $t^{1}$. 
At the next level $i=1$, for the path $t^{1}=(1)$, it is possible to add either a pickup vertex 2 or a delivery vertex 3 corresponding to the pickup vertex 1 . A vertex 4 can't be added because there is no corresponding pickup vertex 2 in the current path $t^{1}=(1)$.

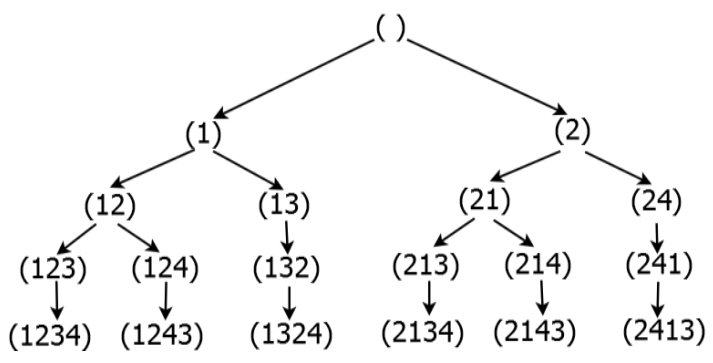

Fig.1. A recursive tree generated by the algorithm

When all full paths have been generated by the algorithm (so we have the complete tree), a resulting path will be a path that owns the best value of the objective function (1).

\section{B. An heuristic solution}

A powerful beam search heuristic [36] can be applied to algorithms that produce recursion tree. The beam search heuristic is an adaptation of the branch and bound method where only some nodes are evaluated in the search tree [37]. At any level, promising nodes are only kept for further branching, and the rest of nodes are being pruned off permanently [37].

In terms of our problem, the beam search heuristic acts at the step of expanding tree nodes (i.e. extending partial paths with points from the set $F^{i}$ ). In an original version, it takes some predefined amount of "best" points from $F^{i}$ (a beam width) for further expanding and pruning off others. We slightly modified heuristic and made the beam width a relative value - some percent of tree nodes. We will call it a relative beam width $(R B W)$.

So, for our problem, the beam search heuristic can be applied as follows:

H1. The algorithm sorts new points from $F^{i}$ by ascending a distance:

- $\quad$ from the depot, if $i=0$ (so, the first point in a route should be as close to the depot as possible);

- a last node in the current path for all other cases.

H2. The GenBase algorithm calls itself recursively only for the first $R B W \%$ of points from $F^{i}(R B W$ is a predefined parameter). Other nodes are pruned off.

H3. Since checking the 3D constraints is the $N P$ complexity task [29], they are checked not when a vehicle arrives to a pickup point, but time-by-time, with a probability check_prob $\in[0 ; 1]$. An only exception is the last level $i=2 n-1$, where we always check the $3 \mathrm{D}$ constraints because it is necessary for the final path to meet them.

The power of the described algorithm and the heuristic is that one can regulate a balance between a solution's quality and the algorithm's operating time by changing the $R B W$ value.

Example 2. Let us demonstrate how the heuristic works for $n=4$ (points 1-4 are pickups and points 5-8 are deliveries). Let's set $R B W=50 \%$ which means that we will expand each tree node with a half of points in $F^{i}$ at all steps $i<2 n-1$.

At the beginning, at the level $i=0, F^{0}$ consists of all pickup vertexes $F^{0}=(1,2,3,4)$. We are sorting them by distances to the depot. Let's assume that we obtain $(2,4,3,1)$, and we take first two points ( 2 and 4$)$ for further expanding. Points 1 and 3 are excluded from further consideration.

The same idea works for the next levels as well. Let's see what's happening at the level 1 for a partial path $t^{1}=(2)$. There're four candidates: three other pickups (1,3 and 4) and a delivery point 6 corresponding to a pickup point $2(6=2+n, n=4)$, so $F^{1}=(1,3,4,6)$. Let's suppose that the points 1 and 6 are the closest ones to the point 2 , so we will expand them by skipping the points 3 and 4. So, here $t^{2}=(21)$ and $t^{2}=(26)$.

In Fig.2, one can see a fragment of the tree for $n=4$, where nodes that were present in $F^{i}$ but later excluded are marked with dots.

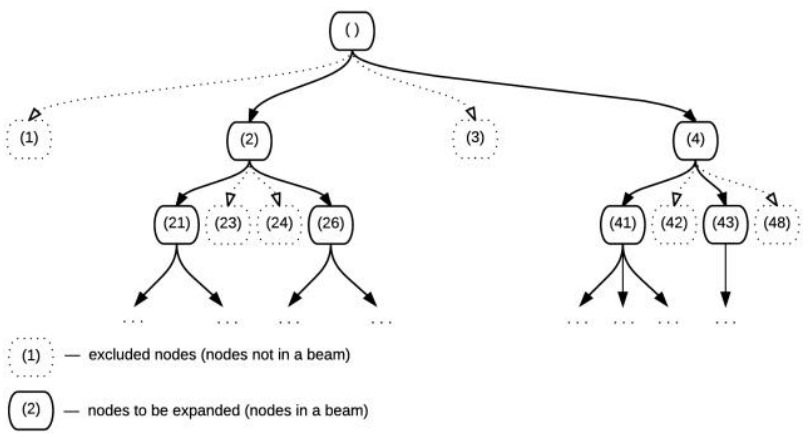

Fig.2. A fragment of the tree for $n=4$

Issue 1. An attentive reader might observe that due to $\mathrm{H} 3$ at a penultimate level $i=2 n-2$, the heuristic can theoretically produce a path $t^{i}$ that doesn't meet the $3 D$ constraints (because $\mathrm{H} 3$ allows skipping a check of the 3D constraints). At the last level, we always check the 3D constraints (to prevent an output of an invalid path), it can be a situation when all paths or partial ones $t^{2 n-2}$ will be invalid (in terms of the 3D constraints) and only a final check at the last level $i=2 n-1$ will let us know that all the current partial paths are invalid.

So, H3 can lead to a situation when the heuristic is not able to give a solution.

An example of this situation is: we have such a small $R B W$ value that only one tree node is expanded at each level (i.e., $R B W=1 \%$ ) and we also have a small check_prob value, so the 3D constraints are rarely checked. So, the algorithm can produce a partial path which doesn't satisfy the 3D constraints and recognize it 
only when it performs a mandatory check of the 3D constraints on the full path. At that point, it can only be seen that the generated path was incorrect.

The described situation can be avoided by increasing values of check_prob and $R B W$. In practice, it is usually enough to set a value of check_prob $=0.2$ to avoid the described situation.

\section{COMPUTATIONAL EXPERIMENTS}

\section{A. A program description}

We developed the program that solves the described problem and has a user-friendly interface.

It is available online at http://pdplitvinenkoapps.rhcloud.com/html/.

Its demo can be found at

https://youtu.be/0vHwssWEUqw.

Input parameters are:

- The vehicle's parameters:

- a vehicle's count (a number of clusters);

- a load area and a weight capacity for every vehicle.

- Parameters of a point:

coordinates of the depot;

a number of the pickup-delivery pairs;

coordinates of each point, a box weight and a size (for pickup points).

- The solution's parameters:

○ $R B W, \%$;

- trans_prob is an indicator that is intended to check the 3D loading constraints for full paths (if unchecked, and trans_prob is 0, the 3D constraints will not be checked, and the solution will be obtained quickly).

The program can:

1) distribute all PDP pairs between vehicles (and form clusters);

2) obtain the heuristic solution described in Section 5.2 for each vehicle (cluster).

\section{B. Comparing the proposed algorithm with well-known} ones

The table below demonstrates results of computational experiments: we obtained a lot of generated paths and a cost of the best path through varying $n$ and $R B W$ (coordinates of the vertexes are generated randomly within the range $0 \ldots 500$ ). A load capacity, a width, a height and a length of the vehicle were set to huge values (so, the 3D constraints were always satisfied).

The results are presented in a format like "a total count of generated paths"/ "a length of the best route". For example, the program generated 298 routes and a cost of the best path was 1869 for $2 n=12$ and $R B W=20 \%$.

We compared the results with the well-known twophase heuristic by Renauld [38] with parameters $R=5$ and various values of $\alpha$ : $\alpha=0.5 ; 1 ; 1.5$ (Tables 1 and 2 ). We programmed the Renauld's algorithm ourselves.

It is worth mentioning that the Renauld's approach does not take into account any loading limits. That is why we mitigated all the loading limits (we assigned large values to $W, H, L$ and $Q$ ) while comparing our results with the Renauld's ones.

As one can see, while our approach is more complex and takes into account the vehicle's loading, the results obtained aren't much worth than the ones obtained by means of a two-phase heuristic. However, a serious disadvantage of our algorithm is its working time: while the Renauld's algorithm is processing data only for a few milliseconds, our program can be processing data for a rather long period (up to 60 seconds for 25 PDP pairs).

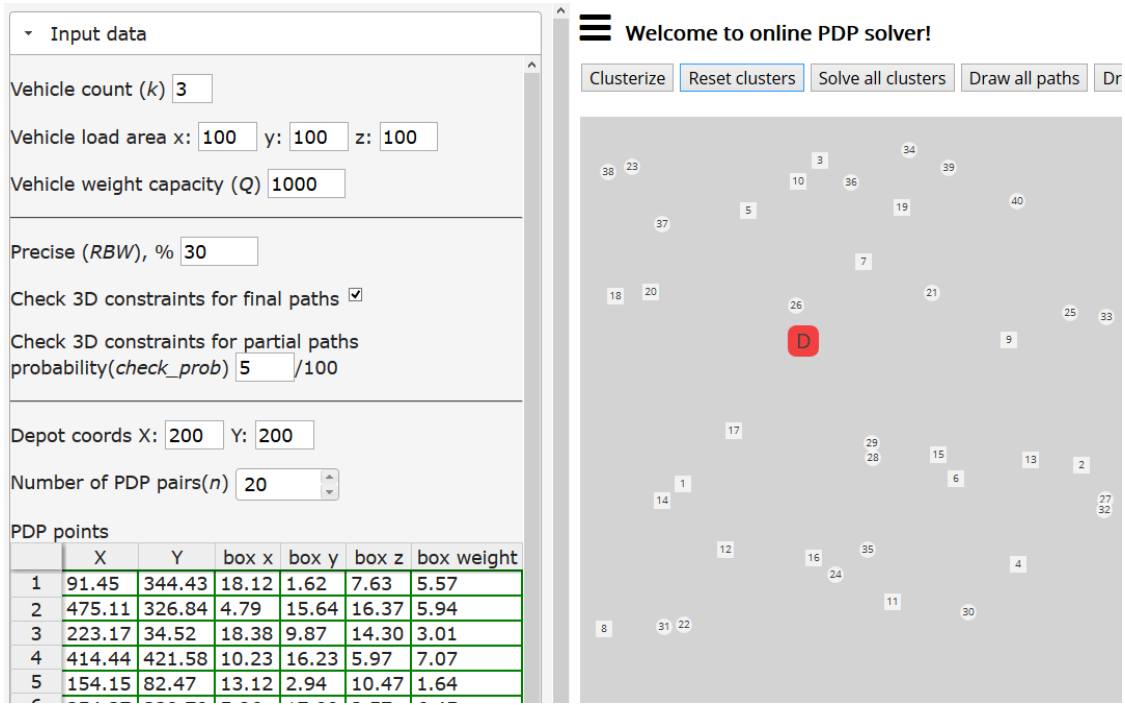

Fig.3. A screenshot with all input parameters and PDP points 


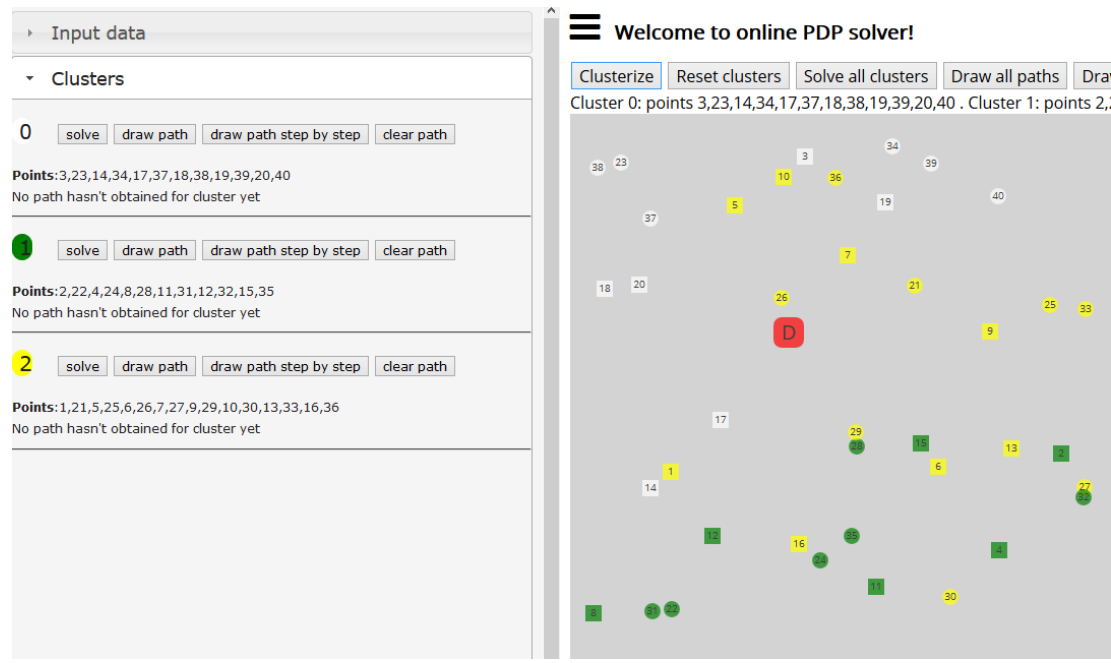

Fig.4. A screenshot of clustering 20 pickup-delivery pairs (squares are pickups and circles are deliveries; the big rounded red square is a depot)
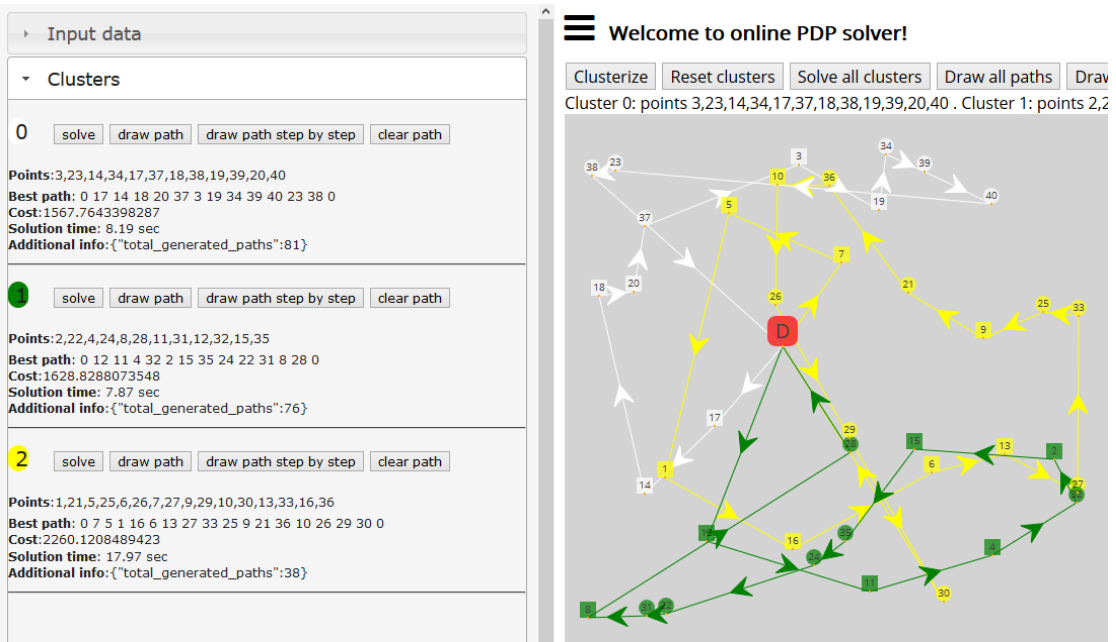

Fig.5. A screenshot of a generated path for each cluster from the example above

Unfortunately, we could not find any articles (except [38]) with publicly accessible test instances that are devoted to the PDP problem with constraints similar to the ones we have considered. That is why we were comparing our results with the Ropke's ones [39], which are shared at http://www.diku.dk/ sropke/.

The Ropke's work is devoted to the multi-vehicle PDP problem with time windows. A formulation of the Ropke's problem also takes into account a capacity $Q$ of every vehicle. While comparing results, we used the same $Q$ value as in the Ropke's samples.

However, our results cannot be clearly matched to the Ropke's ones, since we do not consider time windows

Despite that, we can check that our results are of the same scale compared to the existing ones. In [39], Ropke considers four types of instances (A, B, C, and D) depending on different vehicles' capacities and time windows. We chose $\mathrm{C}$ and $\mathrm{D}$ groups, where time windows are longer: a time window is 120 while a planning horizon is 600 for all vehicles.

In Table 3, one can see the Ropke's results and our results compared to test instances from [39]. We chose $R B W$ values that allowed to get good results in a short time period (less than 25 seconds). We can definitely get a faster or better solution by choosing another $v$ value for every instance.

As we can expect, our total cost is always better than the Ropke's one, because we do not take time windows into account.

Besides [39], the article [25] also solves the PDP problem with the 3D loading constraints, but their problem description and their solution algorithm have too many additional constraints and features we do not have. For example, the article [25]:

- considers reloading of a box so that it can be unloaded and loaded again to another place; our solution algorithm does not have this feature;

- $\quad$ allows multiple boxes to be loaded from a pickup point while we allow only one box in that case;

- considers fragility of a box, so fragile boxes should not be placed under non-fragile ones; we do not have this constraint etc.

That is why we do not see any sense to compare our results with the ones in [25] because our problems and solution algorithms are too different although being similar at the first glance. 
Table 1. Comparison of the Renauld's results and the results obtained by the proposed algorithm (part 1)

\begin{tabular}{|c|c|c|c|c|c|c|c|c|c|c|}
\hline \multirow{2}{*}{$2 n$} & \multirow{2}{*}{$R B W=1 \%$} & \multirow{2}{*}{$R B W=5 \%$} & \multirow{2}{*}{$R B W=10 \%$} & \multirow{2}{*}{$R B W=20 \%$} & \multirow{2}{*}{$20 \%<R B W<50 \%$} & \multirow{2}{*}{$\begin{array}{c}\text { Best } \\
\text { obtained }\end{array}$} & \multirow{2}{*}{$\begin{array}{c}\text { Average } \\
\text { time }\end{array}$} & \multicolumn{3}{|c|}{ Two-phase heuristic } \\
\hline & & & & & & & & Alpha $=0.5$ & Alpha $=1$ & Alpha $=1.5$ \\
\hline 8 & $1 / 2044$ & $1 / 2044$ & $1 / 2044$ & $10 / 1621$ & $180 / 1531$ & 1531 & $<16 \mathrm{~ms}$ & 1656 & 1531 & 1531 \\
\hline 10 & $1 / 1985$ & $1 / 1985$ & $1 / 1985$ & $44 / 1869$ & $1972 / 1664$ & 1664 & $<500 \mathrm{~ms}$ & 1830 & 1750 & 1664 \\
\hline 12 & $1 / 2090$ & $1 / 2090$ & $213 / 1869$ & $298 / 1869$ & $2125 / 1869$ & 1869 & $<500 \mathrm{~ms}$ & 1946 & 1796 & 2099 \\
\hline 14 & $1 / 2198$ & $1 / 2198$ & $1 / 2198$ & $14 / 2148$ & $2666 / 2148$ & 2148 & $<1 \mathrm{~s}$ & 2125 & 2124 & 2267 \\
\hline 16 & $1 / 2520$ & $1 / 2520$ & $130 / 2285$ & $4474 / 2285$ & - & 2285 & $<2 s$ & 2323 & 2228 & 2662 \\
\hline
\end{tabular}

Table 2. Comparison of the Renauld's results and the results obtained by the proposed algorithm (part 2)

\begin{tabular}{|c|c|c|c|c|c|c|c|c|c|}
\hline \multirow{2}{*}{$2 n$} & $v=1 \%$ & $v=5 \%$ & $v=10 \%$ & $v=11 \%$ & \multirow{2}{*}{ Best obtained } & \multirow{2}{*}{$\begin{array}{c}\text { Average } \\
\text { time }\end{array}$} & \multicolumn{3}{|c|}{ Two-phase heuristic } \\
\cline { 8 - 11 } & & & & & & & Alpha=0.5 & Alpha=1 & Alpha=1.5 \\
\hline 20 & $1 / 2404$ & $1 / 2404$ & $3155 / 2097$ & $8921 / 2097$ & 2097 & $<10 \mathrm{~s}$ & 2019 & 2019 & 2035 \\
\hline & $v=1 \%$ & $v=3,7 \%$ & $v=4 \%$ & $v=4,2 \%$ & & & & & \\
\hline 30 & $1 / 3517$ & $41 / 2800$ & $545 / 2650$ & $3102 / 2624$ & 2624 & $<20 \mathrm{~s}$ & 2588 & 2804 & 3295 \\
\hline & $v=1 \%$ & $v=2,7 \%$ & $v=3 \%$ & $v=3,25 \%$ & & & & & \\
\hline 40 & $1 / 3458$ & $170 / 3402$ & $3014 / 3402$ & $9778 / 3222$ & 3222 & $<40 \mathrm{~s}$ & 3526 & 3166 & 3129 \\
\hline & $v=1 \%$ & $v=2 \%$ & $v=2,1 \%$ & $v=2,25 \%$ & & & & & \\
\hline 50 & $1 / 3553$ & $1 / 3553$ & $586 / 3178$ & $6429 / 3151$ & 3151 & $<60 \mathrm{~s}$ & 3036 & 3398 & 3180 \\
\hline
\end{tabular}

Table 3. Comparison of the Ropke's results and the results obtained by the proposed algorithm

\begin{tabular}{|c|c|c|c|c|c|c|}
\hline \multirow{2}{*}{ Ropke's instance } & \multicolumn{2}{|c|}{ Ropke's results } & \multicolumn{4}{c|}{ Our results } \\
\cline { 2 - 7 } & total cost & solution time, $\mathrm{s}$ & total cost & solution time, $\mathrm{s}$ & Routes generated & $R B W, \%$ \\
\hline DD30 & 1133 & 49 & 955 & 0.315 & 16 & 1 \\
\hline DD35 & 1210 & 99 & 1137 & 1.107 & 34 & 2 \\
\hline DD40 & 1352 & 136 & 1198 & 4.253 & 91 & 2 \\
\hline DD45 & 1483 & 132 & 1322 & 20.8 & 348 & 2 \\
\hline DD50 & 1600 & 105 & 1425 & 7.833 & 1165 & 1.7 \\
\hline DD55 & 1743 & 124 & 1518 & 21.684 & 189 & 1.5 \\
\hline DD60 & 1869 & 247 & 1716 & 5.144 & 32 & 1.2 \\
\hline DD65 & 2125 & 209 & 1939 & 4.837 & 23 & 1.1 \\
\hline DD70 & 2220 & 175 & 2184 & 1.786 & 7 & 1 \\
\hline DD75 & 2396 & 201 & 2291 & 2.232 & 7 & 1 \\
\hline CC30 & 1087 & 76 & 1035 & 5.058 & 297 & 3 \\
\hline CC35 & 1230 & 97 & 1172 & 12.823 & 468 & 2.5 \\
\hline CC40 & 1358 & 132 & 1205 & 8.22 & 191 & 2 \\
\hline CC45 & 1509 & 82 & 1404 & 2.065 & 34 & 1.7 \\
\hline CC50 & 1689 & 168 & 1613 & 2.669 & 35 & 1.8 \\
\hline CC55 & 1816 & 196 & 1730 & 12.134 & 108 & 1.3 \\
\hline CC60 & 2015 & 127 & 1823 & 12.111 & 86 & 1.3 \\
\hline CC65 & 2172 & 145 & 2024 & 14.776 & 80 & 1.1 \\
\hline CC70 & 2201 & 288 & 2159 & 11.675 & 49 & 1 \\
\hline CC75 & 2375 & 325 & 2327 & 16.105 & 54 & 0.8 \\
\hline
\end{tabular}

\section{Comparing the exact and heuristic solutions: main results}

We described the algorithm to get the exact and heuristic solutions for a lot of instances. Every instance is a combination of the following input parameters:

- 4 problem sizes: $n=3,4,5$ and 6 ;

- 6 sets of the loading constraints: the load area is a cube with sides $=50 ; 70 ; 90 ; 110 ; 130 ; 150$ and $Q=100 ; 200 ; 300 ; 400 ; 500 ; 600$ respectively;

- 5 sets of points' coordinates, box sizes and weights.
The points' coordinates were randomly chosen from a range $[1 ; 500000]$, the box size was a cube with a side selected from a range $[1 ; 50]$ and the weight was selected from a range $[1 ; 100]$.

For each sample out of $4 \times 6 \times 5=120$ instances, we tried to obtain an exact solution (i.e. a solution for $R B W=100 \%$.) and 3 heuristic solutions for $R B W=10 ; 30 ; 50 \%$. check_prob was 0.2 for all instances.

We were able to get the exact solutions for $n=6$ only for the small 3D constraints (for $Q<90$ ). For $Q>=90$, the exact solution takes at least 3 hours and more than a 
week in most cases. So, we excluded instances with $n=6$ and $Q>=90$ from further consideration.

After obtaining the heuristic solutions, we compared the resulting cost (1) with a cost of the optimal path obtained by the exact solution. We calculated only a relative cost increase (because the cost of the heuristic solution cost is always bigger or equal to the cost of exact solution):

$$
\text { cost_increase }=\frac{h e u-e x}{e x}
$$

where heu denotes the cost (1) of the heuristic solution; ex determines the cost (1) of the exact solution.

We have put to analysis the following issues:

1) how cost_increase depends on $n$ and $R B W<100 \%$ (Fig.6);

2) how the heuristic solution time (in seconds) depends on $n$ and $R B W$ (Fig.7);

3) a relative frequency of the issue 1 occurring for various $n, R B W$ and the loading area cube sizes (Tables 47);

4) a relative frequency of the 'jack pot' when the heuristic produces the optimal solution (the same as the exact one) for various $n$ and $R B W$ (Fig.8)

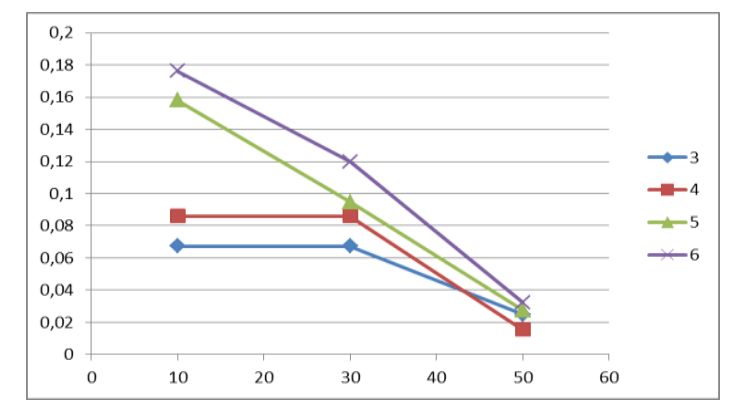

Fig.6. Dependency of cost_increase ( $y$ - axis) on $n(n=3,4,5,6)$ and RBW (x - axis)

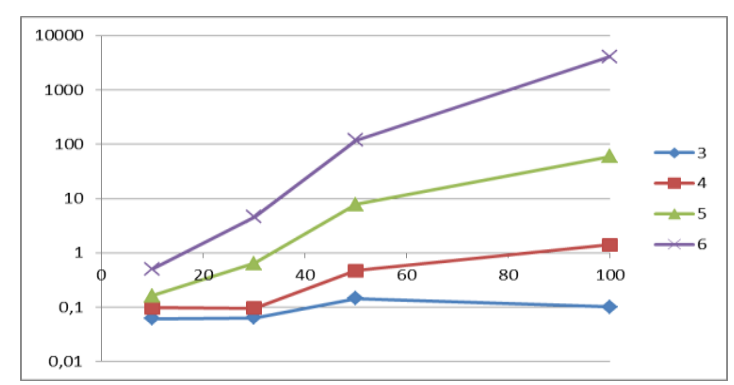

Fig.7. Dependency between n, RBW (x - axis) and the heuristic solution time (y - axis)

Table 4. A relative frequency of the issue 1 occurring for various $R B W$ and the loading area cube sizes $(n=3)$

\begin{tabular}{|c|c|c|c|}
\hline & \multicolumn{3}{|c|}{ Load area cube side } \\
\hline RBW, \% & 50 & 70 & $>70$ \\
\hline 10 & 0,12 & 0 & 0 \\
\hline 30 & 0,12 & 0 & 0 \\
\hline 50 & 0 & 0 & 0 \\
\hline
\end{tabular}

Table 5. A relative frequency of the issue 1 occurring for various $R B W$ and the loading area cube sizes $(n=4)$

\begin{tabular}{|c|c|c|c|}
\hline & \multicolumn{3}{|c|}{ Load area cube side } \\
\hline RBW, \% & 50 & 70 & $>70$ \\
\hline 10 & 0,76 & 0,2 & 0 \\
\hline 30 & 0,7 & 0,2 & 0 \\
\hline 50 & 0,23 & 0 & 0 \\
\hline
\end{tabular}

Table 6. A relative frequency of the issue 1 occurring for various $R B W$ and the loading area cube sizes $(n=5)$

\begin{tabular}{|c|c|c|c|}
\hline & \multicolumn{3}{|c|}{ Load area cube side } \\
\hline RBW, \% & 50 & 70 & $>70$ \\
\hline 10 & 0,58 & 0,2 & 0 \\
\hline 30 & 0,29 & 0,2 & 0 \\
\hline 50 & 0 & 0 & 0 \\
\hline
\end{tabular}

Table 7. A relative frequency of the issue 1 occurring for various $R B W$ and the loading area cube sizes $(n=6)$

\begin{tabular}{|c|c|c|c|}
\hline & \multicolumn{3}{|c|}{ Load area cube side } \\
\hline RBW, \% & 50 & 70 & $>70$ \\
\hline 10 & 0,47 & 0,4 & 0 \\
\hline 30 & 0,11 & 0 & 0 \\
\hline 50 & 0 & 0 & 0 \\
\hline
\end{tabular}

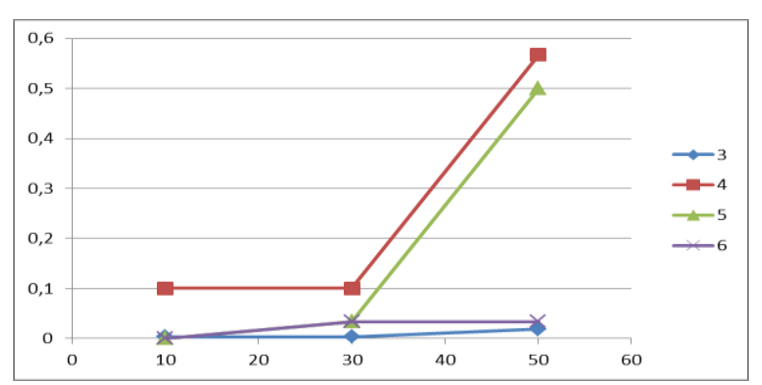

Fig.8. A relative frequency (y - axis) of the 'jack pot' for various RBW $(\mathrm{x}$ - axis) and $\mathrm{n}$

D. Comparing the exact and heuristic solutions: peripheral results

We also generated another set of instances to analyze how the average heuristic solution time depends on check_prob. Similarly to the previous section, each instance is a combination of some input parameters. These parameters are:

- 4 problem sizes: $n=3,4,5,6$;

- 3 sets of the loading constraints: the load area is a cube with sides $=50 ; 75 ; 150$ and $Q=100 ; 300 ; 600$ respectively;

3 sets of points' coordinates, box sizes and weights. The points' coordinates were obtained the same way described in the previous section.

For each sample out of $4 \times 3 \times 3=36$ instances, we obtained the heuristic solutions for various combinations of $R B W=10 ; 30 ; 50 \%$ and check_prob $=0.1 ; 0.2 ; 0.3 ; 0.4$. So, we've got $3 \times 4=12$ heuristic solutions for each instance.

Then we analyzed how the heuristic solution time 
depends on check_prob for various $n$ and $R B W$. Tables below contain the average heuristic solution time (in seconds) for 9 experiments ( 3 sets of the loading constraints $\mathrm{x} 3$ sets of the points' coordinates and box parameters) for the same values of $n$ and $R B W$.

Table 8. check_prob for various $n$ and $R B W(n=3)$

\begin{tabular}{|c|c|c|c|c|}
\hline$n=3$ & \multicolumn{4}{|c|}{ check_prob } \\
\hline RBW & 10 & 20 & 30 & 40 \\
\hline 10 & 0.04 & 0.08 & 0.06 & 0.08 \\
\hline 30 & 0.05 & 0.05 & 0.07 & 0.09 \\
\hline 50 & 0.09 & 0.12 & 0.16 & 0.22 \\
\hline
\end{tabular}

Table 9. check_prob for various $n$ and $R B W(n=4)$

\begin{tabular}{|c|c|c|c|c|}
\hline$n=4$ & \multicolumn{4}{|c|}{ check_prob } \\
\hline RBW & 10 & 20 & 30 & 40 \\
\hline 10 & 0.05 & 0.08 & 0.14 & 0.12 \\
\hline 30 & 0.05 & 0.08 & 0.11 & 0.14 \\
\hline 50 & 0.24 & 0.38 & 0.52 & 0.67 \\
\hline
\end{tabular}

Table 10. check_prob for various $n$ and $R B W(n=5)$

\begin{tabular}{|c|c|c|c|c|}
\hline$n=5$ & \multicolumn{4}{|c|}{ check_prob } \\
\hline RBW & 10 & 20 & 30 & 40 \\
\hline 10 & 0.09 & 0.13 & 0.20 & 0.23 \\
\hline 30 & 0.31 & 0.56 & 0.77 & 0.93 \\
\hline 50 & 3.84 & 6.46 & 9.61 & 12.52 \\
\hline
\end{tabular}

Table 11. check_prob for various $n$ and $R B W(n=6)$

\begin{tabular}{|c|c|c|c|c|}
\hline$n=6$ & \multicolumn{4}{|c|}{ check_prob } \\
\hline RBW & 10 & 20 & 30 & 40 \\
\hline 10 & 0.13 & 0.20 & 0.27 & 0.34 \\
\hline 30 & 1.51 & 2.74 & 3.69 & 4.94 \\
\hline 50 & 42.74 & 75.96 & 105.79 & 135.25 \\
\hline
\end{tabular}

\section{E. Experiments based on large samples}

We launched the heuristic solution on large instances ( $n$ is up to 50) with different $R B W$ values. To speed up the calculation time, we set the $3 \mathrm{D}$ constraints to be always met $(Q=10000$ and the load area is a cube with a side of 5000). Each instance had:

- one of 9 problem sizes: $n=$ $10 ; 15 ; 20 ; 25 ; 30 ; 35 ; 40 ; 45 ; 50$;

- 5 sets of the points' coordinates.

For each sample out of $9 \times 5=45$ instances, we obtained 20 heuristic solutions for $R B W=0.25 ; 0.5 ; \ldots .5 \%$.

We set the solution time limit to 1000 seconds. Fig.9 shows the maximum $R B W$ for where the solution time was less than our time limit. These results can be understood as the maximum precision we can obtain in a short time interval for each $n$.

For the solutions that satisfied the time limit, we analyze how the heuristic solution time (in seconds) depends on $n$ and RBW (Fig.10). Let's notice that we use a logarithmic y-axis in Fig.10 because the solution time varies significantly.

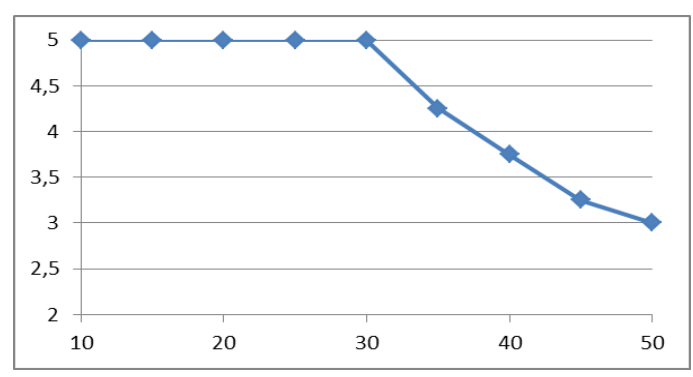

Fig.9. The maximum precision for the solution (RBW, y-axis) we can obtain in a short time period for each $\mathrm{n}$ (x-axis)

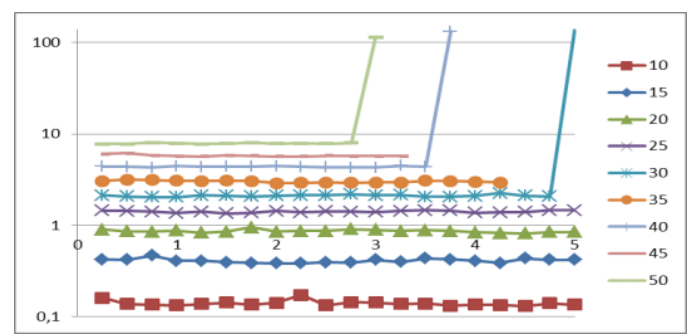

Fig.10. Dependency between $\mathrm{n}$ (see legend), RBW (x - axis) and the heuristic solution time (y - axis)

\section{CONCLUSION}

In this article, the mathematical model for the one-toone Pickup and Delivery Problem with the 3D loading constraints applying the combinatorial configuration concepts instead of Boolean variables was built.

The universal GenBase algorithm was applied to generating PDP paths that satisfy the 3D constraints (to be checked by the algorithm [29We also described how to get the exact solution; at the same time, we described making use of a slightly modified version of the beam search heuristic to obtain the high-quality solution for a feasible time.

Advantages of the proposed algorithm are its ability of balancing between time measurements, a quality of the solution and its flexibility: changing a way of forming the set $F^{i}$ and a way of expanding the solution for tree nodes can help adapt easily the algorithm for being applied to different combinatorial optimization problems. For example, the GenBase algorithm was used for generation of a large number of combinatorial sets [35] as well as for optimization of a linear function in a set of cyclic permutations [40].

\section{REFERENCES}

[1] B. Golden, S. Raghavan, and E. Wasil, The vehicle routing problem: latest advances and new challenges. Springer Science \& Business Media, 2008.

[2] V. Pillac, M. Gendreau, C. Guéret, and A. L. Medaglia, "A review of dynamic vehicle routing problems," European Journal of Operational Research, vol. 225, no. 1, pp. 1-11, Feb. 2013.

[3] S. Kumar and R. Panneerselvam, "A survey on the vehicle routing problem and its variants," Intelligent Information Management, vol. 4, no. 3, p. 66, 2012.

[4] M. Gendreau, M. Iori, G. Laporte, and S. Martello, "A 
Tabu Search Algorithm for a Routing and Container Loading Problem," Transportation Science, vol. 40, no. 3, pp. 342-350, Aug. 2006.

[5] M. Iori and S. Martello, "Routing problems with loading constraints," TOP, vol. 18, no. 1, pp. 4-27, Jul. 2010.

[6] H. Pollaris, K. Braekers, A. Caris, G. K. Janssens, and S. Limbourg, "Vehicle routing problems with loading constraints: state-of-the-art and future directions," $O R$ Spectrum, vol. 37, no. 2, pp. 297-330, Mar. 2015.

[7] S. N. Parragh, K. F. Doerner, and R. F. Hartl, "A survey on pickup and delivery problems," Journal für Betriebswirtschaft, vol. 58, no. 1, pp. 21-51, Apr. 2008.

[8] G. Berbeglia, J.-F. Cordeau, I. Gribkovskaia, and G. Laporte, "Static pickup and delivery problems: a classification scheme and survey," TOP, vol. 15, no. 1, pp. 1-31, May 2007.

[9] J. Cordeau, G. Laporte, and S. Ropke, "Recent models and algorithms for one-to-one pickup and delivery problems," in The vehicle routing problem: latest advances and new challenges, vol. 43, 2008, pp. 327-357.

[10] M. W. P. Savelsbergh and M. Sol, "The General Pickup and Delivery Problem," Transportation Science, vol. 29, no. 1, pp. 17-29, Feb. 1995.

[11] S. Ropke, J.-F. Cordeau, and G. Laporte, "Models and branch-and-cut algorithms for pickup and delivery problems with time windows," Networks, vol. 49, no. 4, pp. 258-272, 2007.

[12] P. Toth and D. Vigo, Vehicle routing: problems, methods, and applications. Society for Industrial and Applied Mathematics, 2014.

[13] W. P. Nanry and J. Wesley Barnes, "Solving the pickup and delivery problem with time windows using reactive tabu search," Transportation Research Part B: Methodological, vol. 34, no. 2, pp. 107-121, Feb. 2000.

[14] H. Li and A. Lim, "A Metaheuristic for the Pickup and Delivery Problem with Time Windows," International Journal on Artificial Intelligence Tools, vol. 12, no. 2, pp. 173-186, Jun. 2003.

[15] H. Lim, A. Lim, and B. Rodrigues, "Solving the pickup and delivery problem with time windows using issqueaky wheelli optimization with local search," AMCIS 2002 Proceedings, p. 317, 2002.

[16] G. Pankratz, "A Grouping Genetic Algorithm for the Pickup and Delivery Problem with Time Windows," $O R$ Spectrum, vol. 27, no. 1, pp. 21-41, Jan. 2005.

[17] Q. Lu and M. M. Dessouky, "A new insertion-based construction heuristic for solving the pickup and delivery problem with time windows," European Journal of Operational Research, vol. 175, no. 2, pp. 672-687, Dec. 2006.

[18] R. Bent and P. Van Hentenryck, "A two-stage hybrid algorithm for pickup and delivery vehicle routing problems with time windows," Computers \& Operations Research, vol. 33, no. 4, pp. 875-893, Apr. 2006.

[19] S. Ropke and D. Pisinger, "An Adaptive Large Neighborhood Search Heuristic for the Pickup and Delivery Problem with Time Windows," Transportation Science, vol. 40, no. 4, pp. 455-472, Nov. 2006.

[20] Y. Nagata and S. Kobayashi, "A Memetic Algorithm for the Pickup and Delivery Problem with Time Windows Using Selective Route Exchange Crossover," in Parallel Problem Solving from Nature, PPSN XI, Berlin, Heidelberg: Springer Berlin Heidelberg, 2010, pp. 536545.

[21] J.-F. Côté, M. Gendreau, and J.-Y. Potvin, "Large neighborhood search for the pickup and delivery traveling salesman problem with multiple stacks," Networks, vol.
60, no. 1, pp. 19-30, Aug. 2012.

[22] G. Erdoğan, J.-F. Cordeau, and G. Laporte, "The pickup and delivery traveling salesman problem with first-infirst-out loading," Computers \& Operations Research, vol 36, no. 6, pp. 1800-1808, Jun. 2009.

[23] A. Malapert, C. Guéret, and N. Jussien, "Twodimensional pickup and delivery routing problem with loading constraints," in First CPAIOR Workshop on Bin Packing and Placement Constraints (BPPC'08), 2008.

[24] E. E. Zachariadis, C. D. Tarantilis, and C. T. Kiranoudis, "The Pallet-Packing Vehicle Routing Problem," Transportation Science, vol. 46, no. 3, pp. 341-358, Aug. 2012.

[25] D. Männel and A. Bortfeldt, "A hybrid algorithm for the vehicle routing problem with pickup and delivery and three-dimensional loading constraints," European Journal of Operational Research, vol. 254, no. 3, pp. 840-858, Nov. 2016.

[26] T. Bartók and C. Imreh, "Pickup and Delivery Vehicle Routing with Multidimensional Loading Constraints," Acta Cybernetica, vol. 20, no. 1, pp. 17-33, 2011.

[27] D. Pisinger and S. Ropke, "A general heuristic for vehicle routing problems," Computers \& Operations Research, vol. 34, no. 8, pp. 2403-2435, Aug. 2007.

[28] G. Scheithauer, Y. G. Stoyan, and T. Y. Romanova, "Mathematical Modeling of Interactions of Primary Geometric 3D Objects," Cybernetics and Systems Analysis, vol. 41, no. 3, pp. 332-342, May 2005.

[29] I. V. Grebennik, A. V. Pankratov, A. M. Chugay, and A. V. Baranov, "Packing n-dimensional parallelepipeds with the feasibility of changing their orthogonal orientation in an n-dimensional parallelepiped," Cybernetics and Systems Analysis, vol. 46, no. 5, pp. 793-802, Sep. 2010.

[30] S. K. Ali, Z. Naser Azeez, and A. Abdul-Hussein Ouda, "A New Clustering Algorithm for Face Classification," International Journal of Information Technology and Computer Science (IJITCS), vol. 8, no. 6, pp. 1-8, 2016.

[31] J. A. Hartigan and M. A. Wong, "Algorithm AS 136: A K-Means Clustering Algorithm," Applied Statistics, vol. 28, no. 1, p. 100, 1979.

[32] A. C. Fabregas, B. D. Gerardo, and B. T. Tanguilig III, "Enhanced Initial Centroids for K-means Algorithm," International Journal of Information Technology and Computer Science (IJITCS), vol. 9, no. 1, pp. 26-33, 2017.

[33] A. Chadha and S. Kumar, "Extension of K-Modes Algorithm for Generating Clusters Automatically," International Journal of Information Technology and Computer Science (IJITCS), vol. 8, no. 3, pp. 51-57, 2016.

[34] Y. Stoyan and I. Grebennik, "Description and Generation of Combinatorial Sets Having Special Characteristics(Bilevel Programming, Optimization Methods, and Applications to Economics)," Biomedical fuzzy and human sciences: the official journal of the Biomedical Fuzzy Systems Association, vol. 18, no. 1, pp. 83-88, 2013.

[35] I. V. Grebennik and O. S. Lytvynenko, "Generating combinatorial sets with given properties," Cybernetics and Systems Analysis, vol. 48, no. 6, 2012.

[36] B. T. Lowerre, "The HARPY Speech Recognition System." Carnegie Mellon University, 1976.

[37] I. Sabuncuoglu and M. Bayiz, "Job shop scheduling with beam search," European Journal of Operational Research vol. 118, no. 2, pp. 390-412, Oct. 1999.

[38] J. Renaud, F. F. Boctor, and J. Ouenniche, "A heuristic for the pickup and delivery traveling salesman problem," Computers \& Operations Research, vol. 27, no. 9, pp. 905-916, Aug. 2000. 
[39] S. Ropke and J.-F. Cordeau, "Branch and Cut and Price for the Pickup and Delivery Problem with Time Windows," Transportation Science, vol. 43, no. 3, pp. 267-286, Aug. 2009.

[40] I. Grebennik, O. Lytvynenko, and O. Titova, "Optimization of linear function on a set of cyclic permutations," Bionics of intellect, vol. 2, no. 67, 2012.

\section{Authors' Profiles}

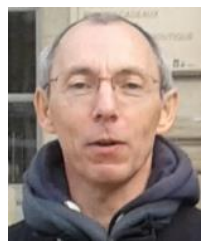

Rémy Dupas was born in 1961. He is a professor at University of Bordeaux. Scientific interests: operational research, combinatorial optimization, industrial engineering, production and transportation problems (scheduling, supply chain planning and vehicle routing).

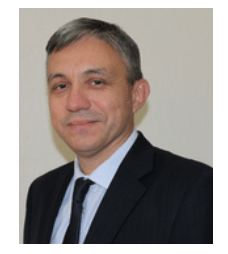

Igor Grebennik was born in 1966. He is D.Sc., professor, Chair of Systems Engineering Department at Kharkiv National University of Radio Electronics.

I.Grebennik is an author of more than 180 publications and eight books.

Scientific interests: Combinatorics, Combinatorial Generation, Combinatorial Optimization, Combinatorial Optimization Problems of Placement of Objects, Mathematical Modeling, Vehicle routing problems.

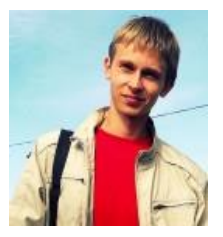

Oleksandr Lytvynenko was born in 1992. He graduated from Kharkiv National University of Radioelectronics (the Faculty of Automatics and Computer Technologies, specialization System Engineering) in 2013. He is currently a Ph.D. student and a web developer.

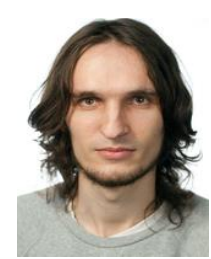

Oleksij Baranov was born in 1984. He graduated from Kharkiv National University of Radioelectronics in 2007. He has got a Ph.D. degree in 2010. Scientific interests: Applied and Computational Mathematics, Simulation and Modelling, Combinatorial Optimization.

How to cite this paper: Rémy Dupas, Igor Grebennik, Oleksandr Lytvynenko, Oleksij Baranov, " An Heuristic Approach to Solving the one-to-one Pickup and Delivery Problem with Three-dimensional Loading Constraints", International Journal of Information Technology and Computer Science(IJITCS), Vol.9, No.10, pp.1-12, 2017. DOI: 10.5815/ijitcs.2017.10.01 